\title{
The Landscape of Targeted Immune Responses in the HIV-1 Vaccine Field
}

\author{
Jeffrey T. Safrit, Georgia D. Tomaras, ${ }^{2}$ Tomáš Hanke, Allan C. deCamp, and Yegor Voronin ${ }^{4}$
}

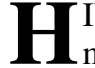
IV-1 VACCINE DEVELOPMENT is rapidly advancing numerous diverse vaccine candidates based on a variety of hypotheses about what constitutes protective HIV-1 immunity. ${ }^{1-3}$ It is common to differentiate vaccine candidates as being antibody-based, T-cell-based, or both, which is a useful classification, but it does not provide enough granularity to capture the multiple hypotheses regarding the contribution of particular immune responses to protection against HIV-1.

We conducted a landscape analysis of the immune responses that are viewed as potentially protective by HIV-1 vaccine researchers (Fig. 1). We interviewed 10 investigators involved in 12 current phase I/IIa studies and asked them to list the immune responses that they wished to elicit with their vaccine candidate(s) divided into the following three categories:

Take: Please list responses that may indicate "take" of the vaccine, but may not by themselves be critical for protection.

Minimal: What are the bare minimum immune responses that would indicate that the vaccine should be advanced to the next level of clinical development?

Optimal: In addition to the responses already listed, are there desired/optimal immune responses that you expect to eventually generate with this candidate (or a version of it)?

We specifically focused on investigators' hypotheses rather than on formal trial endpoints or go/no-go decisions. By capturing individual opinions, we reasoned that the outcome would be less impacted by the need to compromise with collaborators or funders, by product development challenges, and by the calls to allow better cross-trial data comparisons. ${ }^{4}$ We also expected personal opinions to better reflect the latest discoveries in the field.

Although it is difficult to provide a representative response, a couple of examples may be illustrative. For one researcher, appearance of polyclonal binding antibodies to Env was an indication of vaccine take, whereas the tier 2 virus neutralization was the minimal target, with the longterm goals of making humoral responses durable and complementing them with $\mathrm{CD} 8^{+} \mathrm{T}$-cell responses to the virus. Another researcher felt that, as the first step, their vaccine candidate should elicit durable polyclonal binding antibodies that exhibited at least some antiviral activity [tier 1 virus neutralization or antibody-dependent cell-mediated cytotoxicity (ADCC)] together with $\mathrm{CD} 4^{+} \mathrm{T}$-cell responses to Env, whereas the tier 2 virus neutralization was an "optimal" longer term goal.

Frequency distributions of identified responses showed a wide diversity of opinions regarding the potentially protective responses against HIV-1 (Fig. 1A, C). These results are in contrast with the perception that most researchers in the field are targeting the same ideal immune response for their vaccine regimen and they show a healthy and vibrant field, open to exploring multiple competing hypotheses.

We visualized the diversity of the candidate vaccines by calculating pair-wise distances between them based on the similarity of the chosen responses and using those distances to build neighbor-joining trees (Fig. 1B, D). The trees had long branches stemming from points close to the root of the tree and did not exhibit much clustering, especially the "optimal" responses. Still, some groups could be identified, and distinctive immune responses common to those groups are shown.

The majority of the "minimal" desired immune responses were humoral responses, consistent with the field's current focus on antibodies and because recent renewed interest in $\mathrm{CD}^{+}$T-cell-based vaccines ${ }^{5}$ has not yet translated to phase I trials. However, in the "optimal", landscape, cellular responses were much more prevalent, probably reflecting the commonly expressed view that an effective vaccine will require a combination of humoral and cellular immunity.

\footnotetext{
${ }^{1}$ International AIDS Vaccine Initiative, New York, New York.

${ }^{2}$ Duke University, Durham, North Carolina.

${ }^{3}$ University of Oxford, Oxford, United Kingdom.

${ }^{4}$ Vaccine and Infectious Disease Division and Statistical Center for HIV-1/AIDS Research and Prevention, Fred Hutchinson Cancer Research Center, Seattle, Washington.

${ }^{5}$ Global HIV Vaccine Enterprise, New York, New York.

(C) Jeffrey T. Safrit, et al., 2016; Published by Mary Ann Liebert, Inc. This Open Access article is distributed under the terms of the Creative Commons Attribution Noncommercial License (http://creativecommons.org/licenses/by-nc/4.0/) which permits any noncommercial use, distribution, and reproduction in any medium, provided the original author(s) and the source are credited.
} 

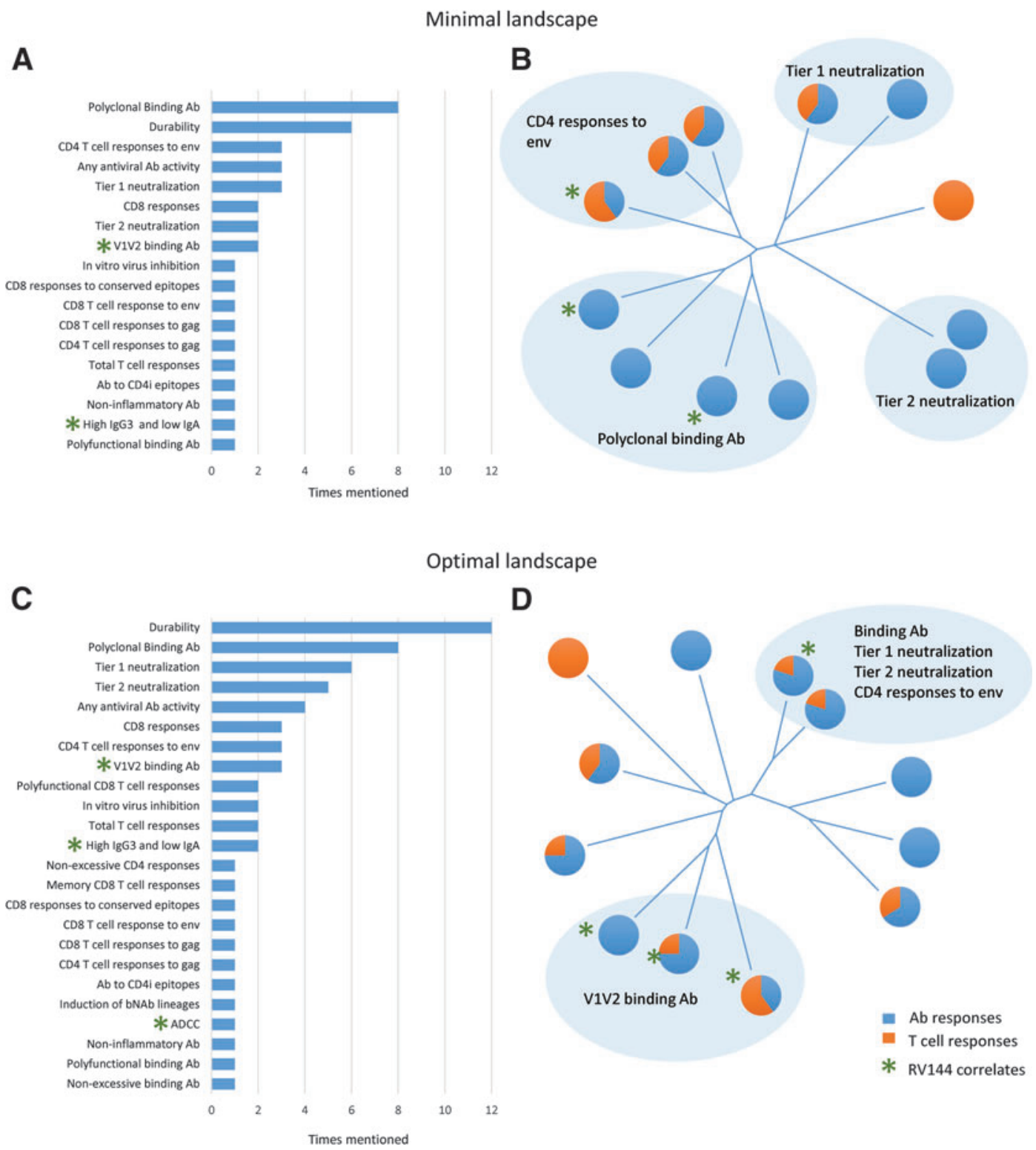

FIG. 1. Immune landscape of candidates based on the minimal (A, B) or the optimal (C, D) desired immune responses. (A, C) show frequency distribution of immune responses identified as desired for the surveyed vaccine candidates. The numbers represent the number of candidates for which a particular response was mentioned. Green stars indicate immune responses that have been previously identified as correlates of risk in the RV144 data analysis. Long-tailed distribution on both graphs reflects that a majority of immune responses were listed for only one or two vaccine candidates. $(\mathbf{B}, \mathbf{D})$ show neighbor-joining trees of vaccine candidates based on the pair-wise distances between candidates as calculated using Canberra distance (6). The pie charts reflect the percentages of humoral and cellular immune responses for each candidate. Blue shaded areas highlight groups of candidates that have indicated immune responses in common with each other and distinct from the rest. Green stars indicate candidates that have among their immune targets at least one immune responses identified as a correlate of risk in the RV144 data analysis. Candidates from the RV144 follow-up program (P5) have not been included in the survey.

In summary, the visualization of the data from this survey provides unique insights into the current hypotheses and strategies driving the HIV-1 vaccine field. Similar approaches could be used to illustrate the diversity or similarity of efforts at other stages of research and development, to analyze candidate portfolios supported by major funders, or to track the evolution of views in the field over time. For example, it would be informative to see whether, several years from now, a similar survey showed convergence of opinions on what provides protection or whether new immune responses appear on the immunological landscape. Although further work utilizing computational methods to compare existing preclinical and clinical vaccine evaluation data is needed to help identify vaccine candidates that demonstrate unique immunological profiles, we believe this analysis could help future strategic planning in the field. 


\section{Acknowledgments}

We would like to thank the following researchers for agreeing to participate in this survey: Drs. Timothy Fouts, Barney Graham, Barton F. Haynes, Sarah Joseph, Shan Lu, Nelson L. Michael, Harriet L. Robinson, Eric Sandstrom, and Yiming Shao.

\section{Author Disclosure Statement}

No competing financial interests exist.

\section{References}

1. Mann JK, Ndung'u T: HIV-1 vaccine immunogen design strategies. Virol J 2015;12:3.

2. Fauci AS, Marston HD: PUBLIC HEALTH. Toward an HIV vaccine: A scientific journey. Science 2015;349:386387.

3. Corey L, Gilbert PB, Tomaras GD, et al.: Immune correlates of vaccine protection against HIV-1 acquisition. Sci Transl Med 2015;7:310rv7.
4. Manrique A, Adams E, Barouch DH, et al:: The immune space: A concept and template for rationalizing vaccine development. AIDS Res Hum Retroviruses 2014;30:1017-1022.

5. Hansen SG, Ford JC, Lewis MS, et al.: Profound early control of highly pathogenic SIV by an effector memory Tcell vaccine. Nature 2011;473:523-527.

6. R Core Team. R: A Language and Environment for Statistical Computing. Vienna, Austria: R Foundation for Statistical Computing, 2015. Available at www.R-project.org, accessed July 20, 2016.
Address correspondence to: Yegor Voronin Global HIV Vaccine Enterprise 64 Beaver Street No. 352 New York, NY 10004 E-mail: yvoronin@vaccineenterprise.org 